\title{
Controlled deposition of sol-gel sensor material using hemiwicking
}

Mikkelsen, Morten Bo Lindholm; Marie, Rodolphe; Hansen, Jan H; Wencel, Dorota; McDonagh, Colette; Nielsen, Hans Ole; Kristensen, Anders

Published in:

Journal of Micromechanics and Microengineering

Link to article, DOI:

10.1088/0960-1317/21/11/115008

Publication date:

2011

Document Version

Publisher's PDF, also known as Version of record

Link back to DTU Orbit

Citation $(A P A)$ :

Mikkelsen, M. B. L., Marie, R., Hansen, J. H., Wencel, D., McDonagh, C., Nielsen, H. O., \& Kristensen, A. (2011). Controlled deposition of sol-gel sensor material using hemiwicking. Journal of Micromechanics and Microengineering, 21(11), 115008. https://doi.org/10.1088/0960-1317/21/11/115008

\section{General rights}

Copyright and moral rights for the publications made accessible in the public portal are retained by the authors and/or other copyright owners and it is a condition of accessing publications that users recognise and abide by the legal requirements associated with these rights.

- Users may download and print one copy of any publication from the public portal for the purpose of private study or research.

- You may not further distribute the material or use it for any profit-making activity or commercial gain

- You may freely distribute the URL identifying the publication in the public portal 
Controlled deposition of sol-gel sensor material using hemiwicking

This article has been downloaded from IOPscience. Please scroll down to see the full text article.

2011 J. Micromech. Microeng. 21115008

(http://iopscience.iop.org/0960-1317/21/11/115008)

View the table of contents for this issue, or go to the journal homepage for more

Download details:

IP Address: 192.38.67.112

The article was downloaded on 07/11/2011 at 09:45

Please note that terms and conditions apply. 


\title{
Controlled deposition of sol-gel sensor material using hemiwicking
}

\author{
Morten Bo Mikkelsen ${ }^{1}$, Rodolphe Marie ${ }^{1}$, Jan H Hansen ${ }^{2}$, \\ Dorota Wencel $^{3}$, Colette McDonagh ${ }^{3}$, Hans Ole Nielsen ${ }^{2}$ and \\ Anders Kristensen ${ }^{1}$ \\ ${ }^{1}$ DTU Nanotech, Technical University of Denmark, DK-2800 Kgs. Lyngby, Denmark \\ 2 DELTA, DK-2970 Hørsholm, Denmark \\ ${ }^{3}$ Optical Sensors Laboratory, National Centre for Sensor Research, School of Physical Sciences, Dublin \\ City University, Collins Avenue, Glasnevin, Dublin 9, Ireland \\ E-mail: anders.kristensen@nanotech.dtu.dk
}

Received 1 June 2011, in final form 16 August 2011

Published 5 October 2011

Online at stacks.iop.org/JMM/21/115008

\begin{abstract}
Optical sensors are fabricated by depositing liquid sol-gel sensor material on a polycarbonate surface, which has been decorated with arrays of periodic micropillars. Using the principle of hemiwicking, the liquid material is spread, guided by the surface structures, to homogeneously fill the volume between the surface structures and form a liquid film with a thickness determined by the height of the micropillars. After evaporation of solvents, a uniform layer of sensor material resides on the surface. This fabrication method enables easy and reproducible deposits of isolated spots of different sensor materials of precise thickness to be made on plastic surfaces, and it provides an improved method for fabricating cheap optical sensors integrated in disposable lab containers.
\end{abstract}

(Some figures in this article are in colour only in the electronic version)

\section{Introduction}

In reagent-based optical sensing systems, a change in the optical response of an intermediate agent, e.g., an analytesensitive dye molecule, is used to monitor the analyte concentration. Such reagent dyes can be immobilized in a solid matrix of sol-gel silica that is permeable to the analyte, thus making an optical sensor material. In a liquid form, solgel-based materials can be coated on a wide range of substrates, which makes this kind of optical sensor materials very versatile [1].

In biotech applications such as, e.g., cell culturing [2], constant monitoring of $\mathrm{pH}$ and dissolved oxygen is of great importance. Sol-gel-based sensor materials for measuring these analytes [3,4] enable fabrication of cheap optical sensors, which can be integrated in disposable lab ware, such as cultivation bags, shake flasks and petri dishes. Using integrated sensors, invasive probes are avoided and sources of contamination are reduced, since the sensors are disposable and optically read from the outside of the container.
Coatings of sol-gel material can be produced using dip-, spray-, or spin-coating [5-7], and homogeneous thin films of sol-gel sensor material, which are required for obtaining fast and uniform response times of the optical sensors, have been demonstrated on dip-coated substrates [4]. However, for deposition of sensor material on the inside of containers, or when several different sensor materials are to be deposited in separate areas on the same surface, these coating methods are not feasible. In present commercial applications of this sensor technology ${ }^{4}, 5$ patches with sensor material are fabricated and manually glued to the inside of containers.

We present a method for deposition of liquid sensor material on a homogeneous layer in a well-defined region of a surface. By decorating the surface with periodic micropillars and using the principle of hemiwicking [8], a drop of liquid material that is deposited on the microstructured surface will spread, guided by the structures, to homogeneously fill the
4 PreSens Precision Sensing GmbH, Regensburg, Germany. http://www.presens.de
5 Sartorius AG Goettingen, Germany. http://www.sartorius- mechatronics.com 
volume between the structures. Spreading of the liquid is governed by the geometry of the microstructures and surface energy, and the thickness of the deposited film is determined by the height of the micropillars and is thus independent of the volume of the deposited drop. This enables easy and reproducible deposits of spots of the sol-gel sensor material of precise thickness to be made on plastic surfaces.

Microstructuring of the inside of blow-molded plastic containers may be performed using step-and-stamp imprint lithography [9], and for plastic components produced by injection molding, microstructures can be integrated directly in the mold [10]. Both of these fabrication methods are suited for large-scale industrial production.

Spreading by hemiwicking thus enables direct, controlled deposition of spots of the sol-gel sensor material inside containers, and it simplifies the fabrication of optical sensors in disposable lab ware.

\section{Hemiwicking of sol-gel material}

Hemiwicking is the phenomenon of liquid being imbibed and spread by a surface texture, and it has previously been studied in relation to wetting of surfaces [8, 11, 12] and controlled spreading of liquids $[13,14]$. In this paper, we use hemiwicking to produce films of liquid sol-gel sensor material with well-defined thickness. When deposited on a surface, the sol-gel material is a liquid mixture of solvents, i.e. water and ethanol, and solutes. After evaporation of the solvents, the solid-gel matrix, containing the analyte-sensitive dye molecules, remains deposited between the microstructures.

When a drop of liquid is deposited on top of a microstructured surface, some of the liquid will penetrate into the surface texture to wet the microstructures, provided that the equilibrium contact angle between the liquid and surface $\theta_{\text {eq }}<\pi / 2$, while the remaining drop lies as a spherical cap on top of the microstructures. The front of the penetrating liquid will continue to propagate within the surface structure, minimizing surface energy, until it reaches the edges of the drop. However, if $\theta_{\text {eq }}$ is smaller than a critical angle $\theta_{\mathrm{c}}$, as described in [11], hemiwicking is favorable, and the liquid front will continue to spread within the surface structure beyond the extent of the drop. A liquid film is thus formed over the surface, between the microstructures, while the drop is drained. This is sketched in figure $1(a)$ in the case of a surface with a periodic array of micropillars.

Surface structures can pin a contact line, and for disconnected structures like the array of pillars, the liquid front must be activated to continue the progression. For wetting liquids, this is made possible via the meniscus forming around each pillar. In order for the contact line to continue the progression, the meniscus must be able to extend from one row of pillars to the next [12]. Assuming zero mean curvature of the meniscus, the condition for the sufficient extension is $h / l>\tan \theta_{\mathrm{eq}}$ on an array of micropillars of height $h$, radius $r$ and period $d=l+2 r$, as sketched in figure $1(b)$. Before attaining the equilibrium contact angle, the advancing contact line will thus reach the next row of pillars, which will be wetted, and the liquid front will continue to propagate on the

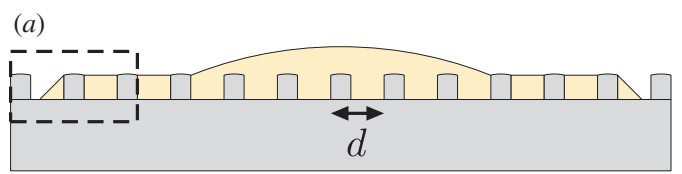

(b) $h / l>\tan \theta_{\mathrm{eq}}$

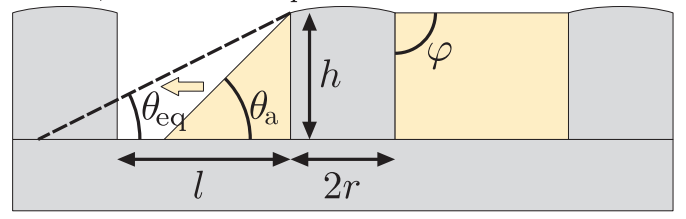

(c) $h / l<\tan \theta_{\mathrm{eq}}$

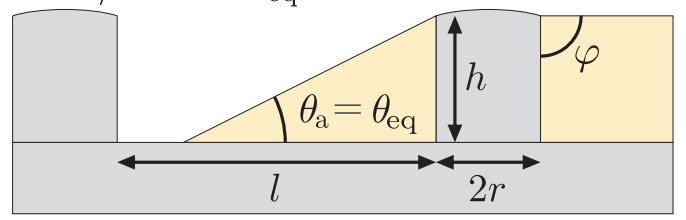

Figure 1. (a) Cross-sectional sketch of a drop of liquid deposited on a two-dimensional array of micropillars of height $h$, radius $r$ and period $d=l+2 r$. Driven by surface energy, a liquid film self-propagates within the microtexture, spreading the liquid and draining the drop, which lies as a spherical cap on top of the pillars. (b) Enlarged view of the dashed box in $(a)$ showing the liquid front in the case of $h / l>\tan \theta_{\mathrm{eq}}$. The contact angle of the advancing liquid front $\theta_{\mathrm{a}}$ is larger than the equilibrium contact angle $\theta_{\mathrm{eq}}$, and the liquid front will reach the next row of pillars before attaining $\theta_{\text {eq }}$. The next row of pillars will thus be wetted, and the propagation continues on the other side. In $(c)$, the same situation is shown for a different geometry with $h / l<\tan \theta_{\text {eq. }}$. In this case, the liquid front is pinned when $\theta_{\mathrm{a}}=\theta_{\mathrm{eq}}$ and the propagation is stopped. At the top of the pillars, the contact line is pinned by the corner, and surface energy forces $\varphi=90^{\circ}$, producing a flat air-liquid interface.

other side. This will proceed until the drop, which was initially on top of the pillars, has been drained completely and all the liquid has been imbibed by the microstructure, provided that the inter-pillar volume is large enough to contain the drop volume.

The contact line at the top of the pillars is pinned by the corner, so that the contact angle $\varphi$ is not limited to $\theta_{\text {eq }}$ but can take a wide range of values $[8,15]$. For narrow structures, surface energy forces $\varphi=90^{\circ}$, producing a flat air-liquid interface [14]. The pillar height $h$ thus exclusively determines the thickness of the deposited liquid layer. The volume of the deposited drop controls the size of the wetted area, but it does not affect the deposited material thickness. Hemiwicking can thus be used for producing liquid films of precise thickness [8].

In the case of a geometry with $h / l<\tan \theta_{\text {eq }}$, as sketched in figure $1(c)$, the liquid front gets pinned when the advancing contact angle $\theta_{\mathrm{a}}$ equals $\theta_{\mathrm{eq}}$, and propagation stops before the next row of pillars is reached [13]. The same situation is found when a spreading liquid reaches the edge of the structured area. However, propagation may continue in other directions on the structured surface, and filling of the surface structure is hence not sensitive to alignment as long as the liquid is deposited inside the patterned area. 
To create a uniform film of sensor material by hemiwicking, it is required that the solvent evaporation of the deposited sol-gel material occurs much slower than the spreading of the material. Otherwise, evaporation during spreading will result in inhomogeneous sensor spots. The propagation speed of the spreading liquid film increases with $h / l$ [13], and hence the geometry of the surface structures can be optimized to minimize the spreading time.

\section{Optimization of surface structures}

In order to find the optimal surface structures for fabricating sensor spots by uniform spreading of sol-gel sensor material, the material was deposited on a series of $7 \mathrm{~mm}$ diameter arrays of pillars of different dimensions and periods, which were fabricated by thermal imprint lithography [16] on a polycarbonate foil. Polycarbonate was chosen since it is a common material for laboratory bottles. Using a Krüss DSA 10 drop shape analyzer, the contact angle of the ETEOSbased sol-gel oxygen sensor material described in [4] was measured to be $\theta_{\mathrm{eq}}=12^{\circ} \pm 3^{\circ}$ on a flat polycarbonate surface. The solvents of the sol-gel material, i.e. water and ethanol, evaporate from a $0.5 \mu 1$ drop in $10 \mathrm{~s}$.

Four-inch silicon stamps with structure heights ranging between 10 and $50 \mu \mathrm{m}$, each containing different square lattice and hexagonal lattice geometries, were fabricated using UV lithography and dry etching. Pillar radii and periods were chosen to make ratios of $h / l$ ranging from 0.06 to 5 . For all geometries, $\theta_{\text {eq }}$ is smaller than $\theta_{\mathrm{c}}$, thus making hemiwicking possible. A fluorosilane coating was applied to the stamp surface by molecular vapor deposition to prevent sticking of the polymer. Imprints were made in $255 \mu \mathrm{m}$ thick sheets of polycarbonate at $170{ }^{\circ} \mathrm{C}$ and $1.3 \mathrm{MPa}$ for $5 \mathrm{~min}$, before cooling the imprint stack to $120^{\circ} \mathrm{C}$ and demolding.

Deposition of the sensor material on the structured polycarbonate foil was performed using an Eppendorf pipette, and best results were obtained by filtering the sensor material with a $0.1 \mu \mathrm{m}$ PTFE syringe filter and deposition in a clean room to avoid dust defects in the coating. After deposition, the sol-gel material was fully dried and condensed by heating to $110^{\circ} \mathrm{C}$ for $18 \mathrm{~h}$.

Figure 2 shows examples of depositions of the sensor material on microstructures of different geometries. Like in [13] and [14], we find that the ability of a given geometry to homogeneously spread the sensor material mainly depends on the ratio of $h / l$, and for structures of equal $h / l$, it is relatively independent of pillar height and lattice pattern, i.e. rectangular or hexagonal. As shown in figure 2, the deposition results can therefore be grouped into four regimes of $h / l$ in relation to the equilibrium contact angle $\tan \theta_{\text {eq }} \approx 0.2$.

- For $h / l<\tan \theta_{\text {eq }}$, the liquid front gets pinned as sketched in figure $1(c)$, and propagation beyond the drop is not possible. Evaporation causes uneven material distribution with ring deposits as described in [17].

- For $\tan \theta_{\text {eq }}<h / l<0.5$, metastable pinning by the pillars happens easily, and propagation is slow and very sensitive to the lattice pattern. This favors formation of polygonal shapes of the deposited material, as described

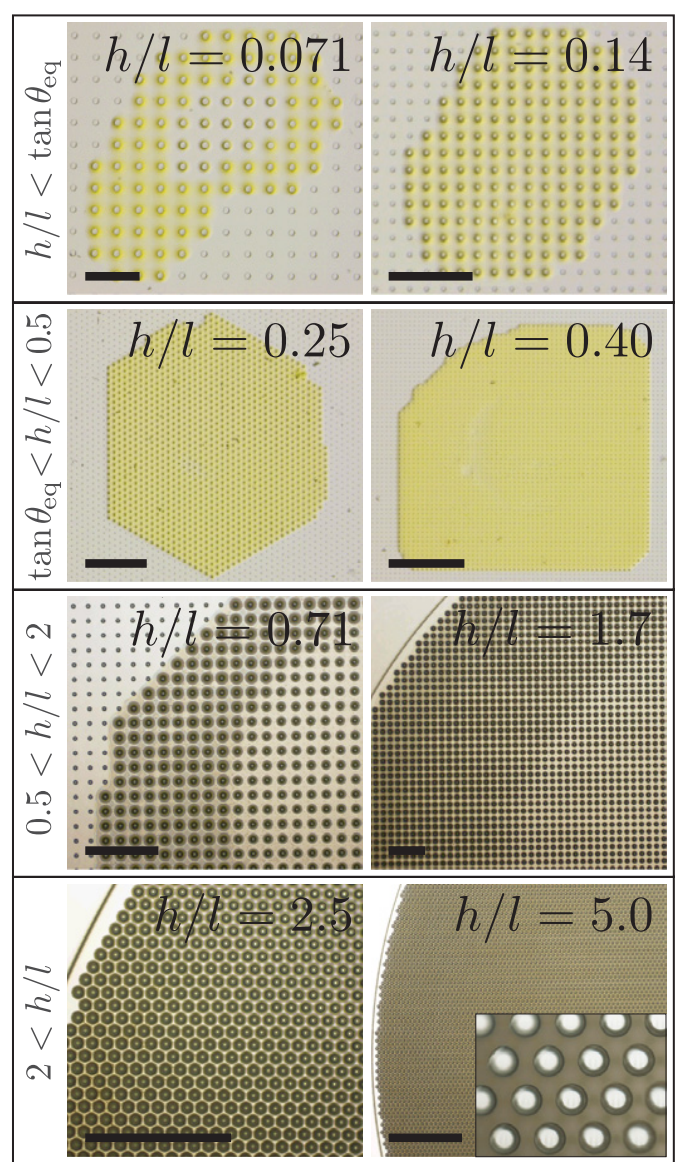

Figure 2. Top view of the sol-gel sensor material deposited on a polycarbonate foil with regions of different pillar geometries. The deposition results are grouped into four regimes, depending on the ratio of $h / l$ in relation to $\tan \theta_{\text {eq }} \approx 0.2$. Homogeneous spreading of the sensor material is only obtained for surface structures with $h / l>2$, as shown in the bottom row. Scale bars show $500 \mu \mathrm{m}$.

in [13]. Unless the deposited volume is small, spreading is incomplete and material is left on top of the surface structures.

- For $0.5<h / l<2$, the deposited material spreads independently of the lattice pattern to form a round deposition area. This is in correspondence with [13] for increased $h / l$. However, the material distribution is uneven, with the deposit being homogeneous at the center but denser at the perimeter. The inhomogeneity diminishes as $h / l$ is increased.

- For $2<h / l$, very homogeneous layers are produced.

Figure 2 shows that the homogeneity of the deposited film increases as the ratio of $h / l$ is increased. We explain this tendency by the increasing propagation speed for increasing $h / l$ [13]. For small $h / l$, the spreading time is large, and much of the solvent evaporates while the material is spreading. The solid content of the liquid material thus increases as the film progresses, resulting in an inhomogeneous deposition. Evaporation-induced redistribution of material, as described in [17], may also play a role. At high $h / l$, the spreading time is small, and less solvent evaporates during spreading. Most of the solvent thus evaporates from the material after a uniform 


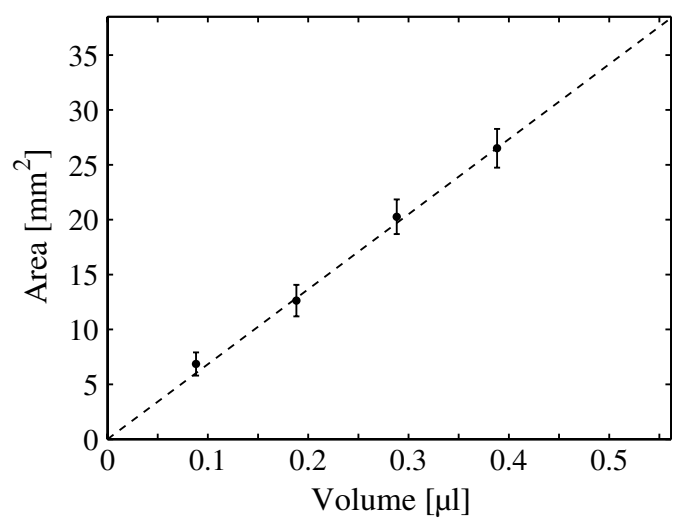

Figure 3. Spreading area as function of volume of oxygen sensor material deposited on a polycarbonate foil with the optimized surface microstructures. Deposition is performed using a $0.1 \mu \mathrm{l}-2.5 \mu \mathrm{l}$-range Eppendorf pipette. The data are corrected for capillary filling of the pipette tip, which added approximately $0.09 \mu \mathrm{l}$ to the volume reading on the pipette. Error bars show standard deviation. The dashed line shows the expected spreading area $A=V /\left(h\left[1-\phi_{\mathrm{S}}\right]\right)$, where $V$ is the deposited volume, $\phi_{\mathrm{S}}=2 \pi r^{2} /\left(\sqrt{3} d^{2}\right), h=23 \mu \mathrm{m}, r=9.5 \mu \mathrm{m}$ and $d=30 \mu \mathrm{m}$. Axis limits correspond to the volume/area of the microstructures of each sensor spot.

film has been made. Uniform evaporation rates over the film result in homogeneous material deposition, considering length scales larger than period $d$ of the pillars. Between the pillars, on length scales smaller than $d$, non-uniform evaporation may cause redistribution of material [17].

Evaporation time depends on the volatility of the solvents and the thickness of the liquid film, and spreading time depends on the distance, contact angle and liquid viscosity. The mentioned regimes of $h / l$ are therefore only valid for the specific structures and materials.

Based on the uniformity of the depositions of sensor material on different surface structures and the sensing performance of the fabricated sensor spots, the best results were obtained with a hexagonal lattice with $h=23 \mu \mathrm{m}$, $r=9.5 \mu \mathrm{m}, d=30 \mu \mathrm{m}$ and $h / l=2.1$. This structure is further characterized in the following sections.

\section{Characterization of material deposition}

In figure 3 , the spreading area is plotted as a function of four different volumes of the sensor material deposited on the optimized microstructure. The linear relation between volume and area shows that spreading of the liquid only occurs as long as the deposited drop can be drained, and the filling stops once all liquid is inside the pillar volume. The excellent agreement between experimental and theoretical spreading areas indicates that the liquid film is uniformly distributed with a film thickness controlled by the pillar height.

If the volume of the deposited sensor material cannot be contained in the inter-pillar volume of a sensor area, the excess material will not spread but stay where deposited on top of the microstructures, thus producing a non-uniformity. Deposited volumes of the sensor material must, therefore, not exceed
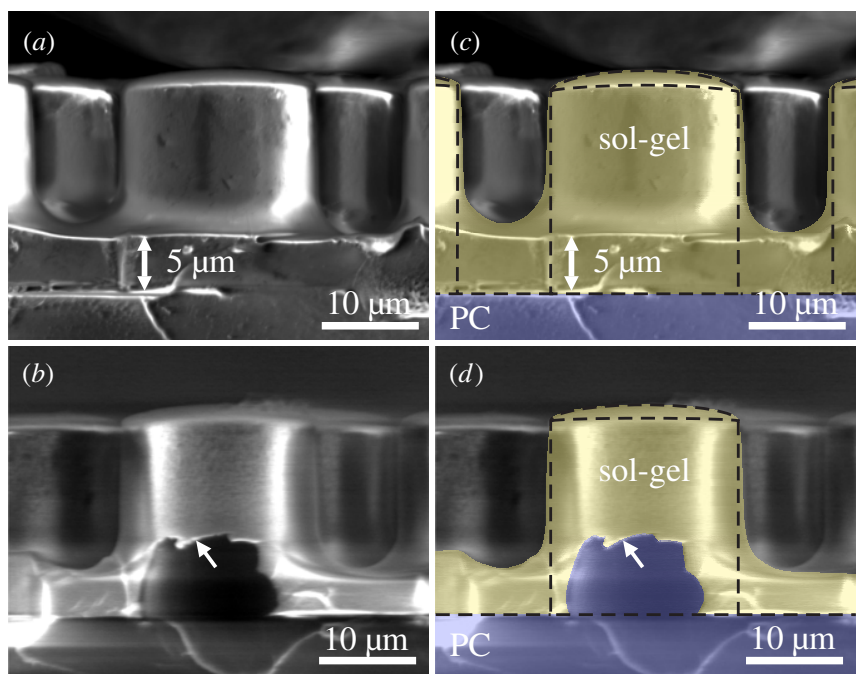

Figure 4. $(a)$, (b) Cross-sectional SEM images of condensed sol-gel oxygen sensor material deposited on $23 \mu \mathrm{m}$ high, $19 \mu \mathrm{m}$ wide pillars imprinted in polycarbonate (PC) foil. (c) and (d) show same images as (a) and (b), but with overlaid colors to highlight the PC substrate and pillars (blue) and the sol-gel coating (yellow). Dashed lines show outline of the front row of pillars. (a), (c) After evaporation of solvents, the thickness of the deposited sensor material is $5 \mu \mathrm{m}$. Between the pillars, menisci are seen. (b), (d) The fracture shows that a thin film of sensor material has been deposited on the vertical side walls of the pillars, indicated by the arrow. Due to the ductility of the PC foil, cleavage was difficult and could only be performed during immersion in liquid nitrogen.

the $0.56 \mu \mathrm{l}$ required to completely fill the $7 \mathrm{~mm}$ diameter microstructured area.

Figure 4 shows cross-sectional SEM images of the condensed sol-gel sensor material on the $23 \mu \mathrm{m}$ high and $19 \mu \mathrm{m}$ wide polycarbonate structures. The thickness of the sensor material is measured to be $\ell=5 \mu \mathrm{m}$ at the center between the pillars. It is seen that the vertical sidewalls have been coated by a layer of sensor material with a thickness of the order of a few hundred nanometers, and at the bottom between the pillars, the surface is meniscusshaped as sketched in figure 5. The material thickness is thus inhomogeneous within each unit cell of the pillar array. However, this inhomogeneity is repeated in every unit cell, so when averaging over length scales larger than the period $d=30 \mu \mathrm{m}$, the material distribution can be considered homogeneous.

For thin films, fluorescence intensity is proportional to material thickness [18], and measurements of the fluorescence intensity of the sensor material were utilized to get a qualitative evaluation of the thickness homogeneity. Fluorescence images recorded with a CCD camera make it possible to evaluate the homogeneity on different length scales.

Fluorescence images were recorded using a Nikon Eclipse inverted fluorescence microscope, equipped with a metal halide light source and a Photometrics-cooled EMCCD camera, and a Nikon TRITC filter cube was used to isolate the fluorescence signal at $620 \mathrm{~nm}$ [19] of the Ru(II)-tris(4,7diphenyl-1,10-phenanthroline) in the oxygen sensor material. A $4 \times$ objective with numerical aperture of 0.13 was used for 

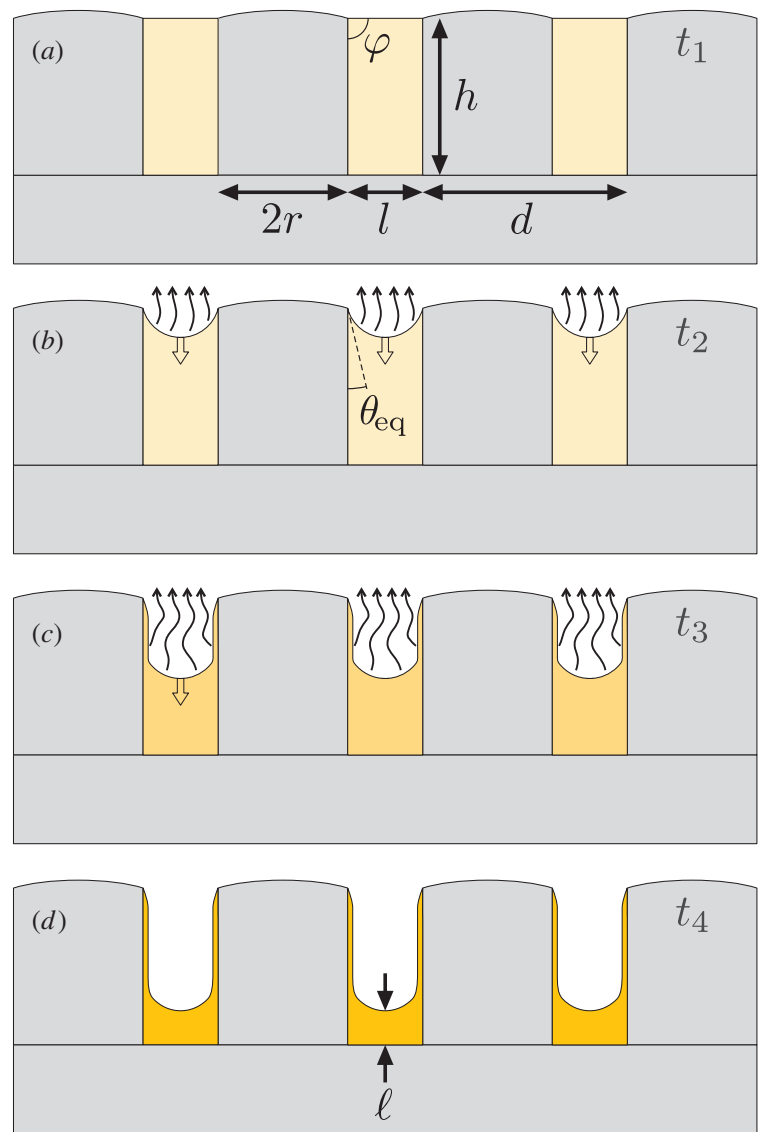

Figure 5. (a) During filling of the microstructure, the air-liquid interface is flat, as sketched in figure 1 . When the solvent evaporates, the decreasing liquid level moves the contact line away from the top corner of the pillar and forces the contact angle to $\theta_{\mathrm{eq}}$, thus creating the meniscus sketched in $(b)$. (c) As the meniscus descends, a thin film is deposited on the vertical sidewalls of the pillars like in a dip-coating process [5]. The solid content of the liquid increases, as indicated by the darker color. $(d)$ The meniscus-shaped surface is maintained while the solid phase of the sensor material condensates and dries completely at the bottom of the pillars.

obtaining a large field of view and large depth of focus, which is convenient for imaging curved surfaces.

On each sensor spot, five fluorescence images were taken, as shown in the top panel of figure 6 . The bottom panel of figure 6 shows intensity histograms of the images $(a)-(e)$. The narrow peak centered at intensity 9.20 au comes from the pillars (dark), where no sensor material is present and only the auto-fluorescence of the polycarbonate substrate produces a signal. This peak thus serves as a reference signal, since it is independent of the deposited sensor material. The much broader peak centered around intensity 13.70 au comes from the fluorescence of the sensor material (bright). Broadening of the peak at intensity 13.70 au is caused by the thickness variation within each unit cell, produced by the conformal deposition seen in figure 4 , but also by inhomogeneity of the light source as well as defects and dust particles on both sides of the substrate. However, particle defects will not displace the peak, due to their small area, but only decrease the peak height.
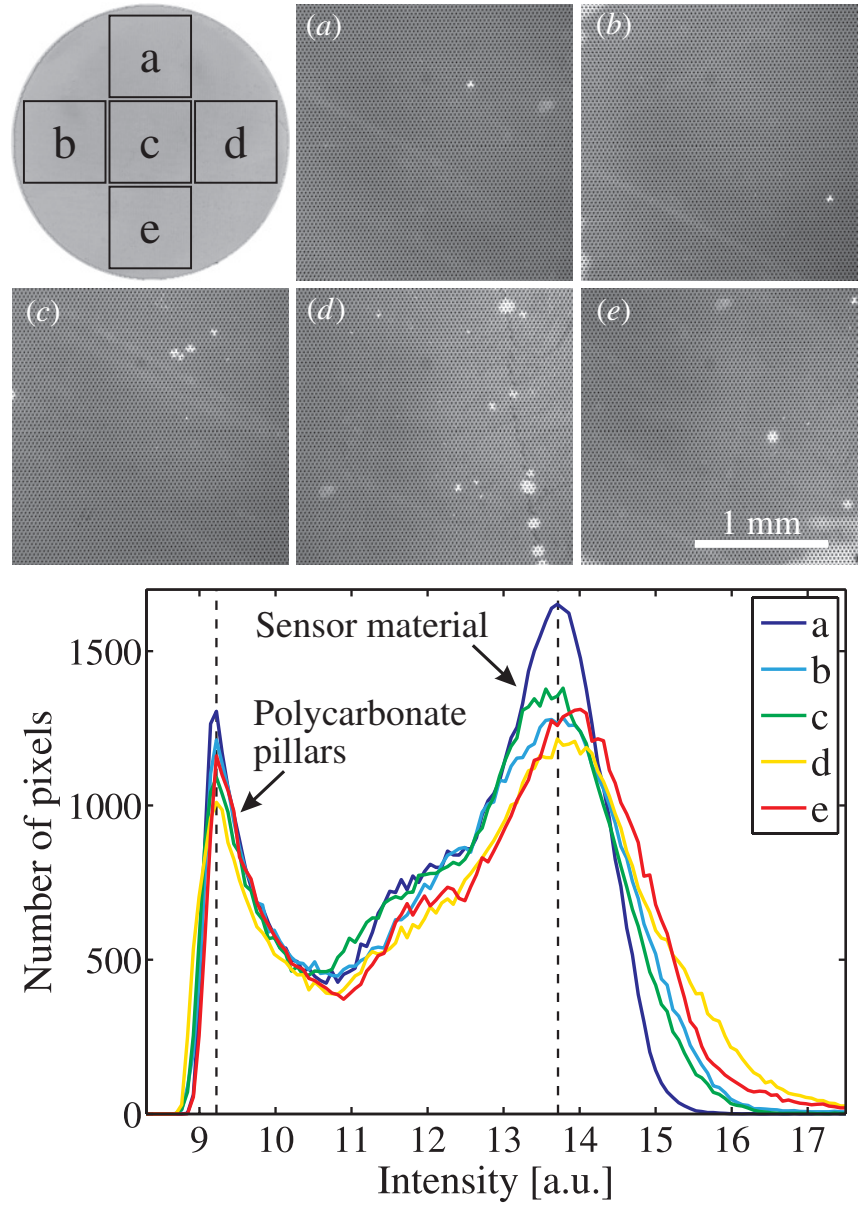

Figure 6. Top panel shows five fluorescence images $(a)-(e)$ and the positions on the sensor spot where they were recorded. Lower panel shows intensity histograms of the five fluorescence images. Average peak centers are indicated by vertical dashed lines at intensities $9.20 \mathrm{au}$ (polycarbonate pillars) and $13.70 \mathrm{au}$ (sensor material).

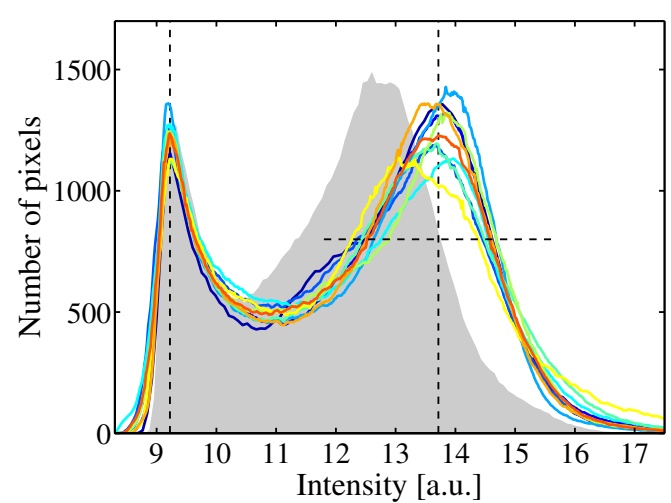

Figure 7. Average histograms of ten different sensor spots. Vertical dashed lines indicate average peak positions at intensities $9.20 \mathrm{au}$ and $13.70 \mathrm{au}$. The gray shaded area shows the average histogram of a sensor spot with a $4 \mu \mathrm{m}$ layer of sensor material, which is produced by $20 \%$ dilution of the sensor material in ethanol. horizontal dashed line at 800 pixels marks the level at which the peak widths in figure 8 are evaluated.

All histograms in figure 6, which were taken at different locations on the same sensor spot, are centered at intensity 


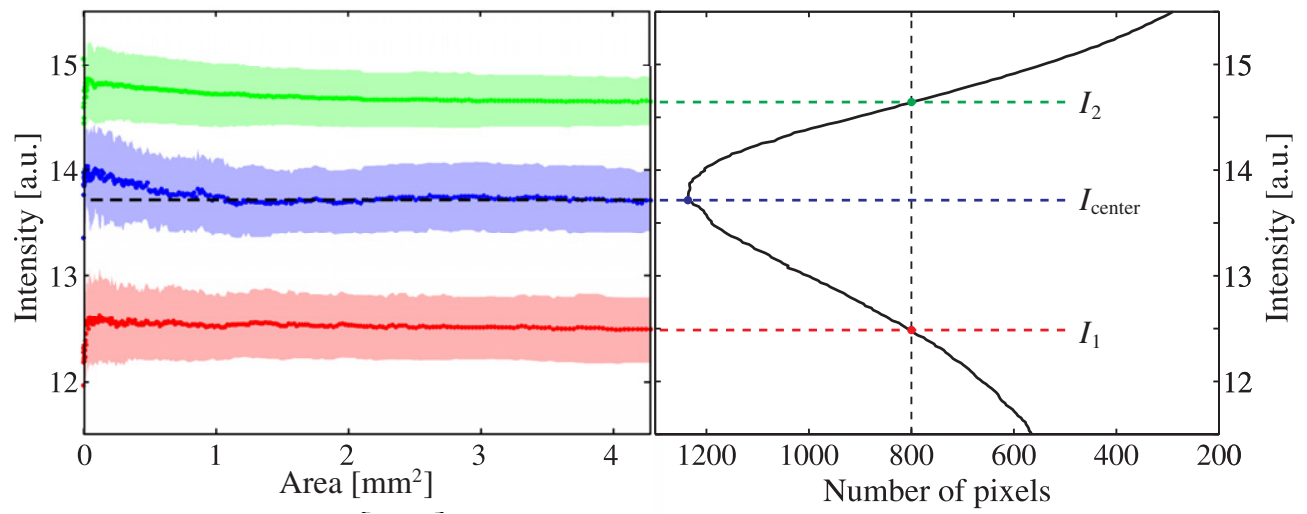

Figure 8. For fluorescence images of ten different sensor spots, histograms were produced for centered image sections of increasing area; $4 \times 4$ pixels, $6 \times 6$ pixels, $8 \times 8$ pixels, and so forth up to the full $512 \times 512$ pixels in each image. For every histogram, the peak center (blue), and the lower peak limit (red) and the upper peak limit (green) at the level of $0.3 \%$ of the pixels, corresponding to the 800 pixels level shown in the right panel, were calculated. In the left panel, the average positions of the peak center and peak limits are plotted as a function of the image section area, and the graph thus shows the evolution of the average position of the fluorescence intensity peak as larger and larger areas are evaluated. The right panel shows the average fluorescence peak based on the full images of ten different sensor spots. Dashed lines at $I_{\text {center }}=13.70$ au, $I_{1}=12.49$ au and $I_{2}=14.64$ au show corresponding levels of the two panels. Shaded areas show the standard deviation.

$13.70 \pm 0.29 \mathrm{au}$. This implies that variations in the thickness distributions are equally small for all five areas within the same spot, and that the deposited material is uniformly spread over the structured surface.

Figure 7 shows average histograms of ten different sensor spots, each curve based on the average of five fluorescence images as in figure 6 . The gray shaded profile shows the average histogram of a sensor spot with $80 \%$ sensor material in ethanol, producing a $4 \mu \mathrm{m}$ layer thickness between the pillars. For comparison, it thus shows how much the intensity histogram changes when the layer thickness is reduced by $1 \mu \mathrm{m}$. The variation between the ten different sensor spots is much smaller and shows a good spot-to-spot reproducibility.

To investigate the influence of the image size on the histograms, for fluorescence images of ten different sensor spots, histograms were produced for centered image sections of increasing size: $4 \times 4$ pixels, $6 \times 6$ pixels, $8 \times 8$ pixels, and so forth up to the full $512 \times 512$ pixels in each image. For each histogram, the peak center and the width, at the level of $0.3 \%$ of the pixels, corresponding to the 800 pixels level shown in figure 7 , were calculated. Figure 8 shows the average peak center and width for the ten spots as a function of the image size. The peak position and width are seen to be constant down to image areas of $0.5 \mathrm{~mm}^{2}$. Below this area, the increasing trend of the peak center, but constant standard deviation, may be caused by light-source inhomogeneity increasing the excitation light intensity toward the image center. Large deviations from the average and increasing standard deviation are only seen for areas below $0.05 \mathrm{~mm}^{2}$, corresponding to approximately 50 unit cells of the pillar geometry. Figure 8 thus shows that, at least above $0.5 \mathrm{~mm}^{2}$, the same intensity histogram is obtained irrespective of the image size, and this self-similarity is another indication of good uniformity of the coating. Furthermore, it shows that the fluorescence intensity does not depend on the area, and that reproducible results can be obtained from small exposed

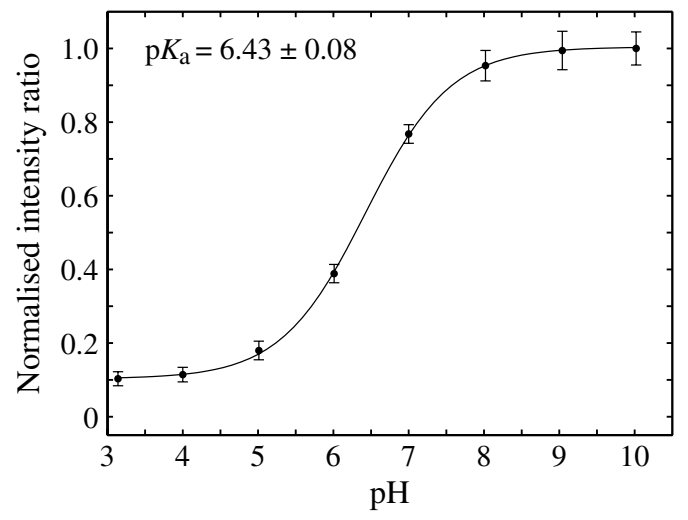

Figure 9. Calibration plot based on five different sensor spots. The normalized intensity ratio between fluorescence at 460 and $408 \mathrm{~nm}$ $\left(I_{460 \mathrm{~nm}} / I_{408} \mathrm{~nm}\right)$ is plotted as a function of $\mathrm{pH}$. Error bars show the standard deviation.

areas. From a practical point of view it means that reproducible measurements of fluorescence can be obtained with a small probe, e.g., a laser, pointed randomly on the much larger sensor spot.

\section{Sensor characterization}

Sensor spots for $\mathrm{pH}$ and oxygen sensing were fabricated by depositing the two different sensor materials on a polycarbonate foil with the optimized $7 \mathrm{~mm}$ diameter pillar arrays of $h=23 \mu \mathrm{m}, r=9.5 \mu \mathrm{m}$ and $d=30 \mu \mathrm{m}$. The performance of the sensors was evaluated as described in [3] and [4].

Five $\mathrm{pH}$ sensor spots were characterized by measuring the intensity ratio between fluorescence at 460 and $408 \mathrm{~nm}$ wavelengths as a function of $\mathrm{pH}$. The result is shown in figure 9 where a sigmoidal function has been fitted to calculate the $\mathrm{p} K_{\mathrm{a}}$ of $6.43 \pm 0.08$. This value corresponds well to the $\mathrm{p} K_{\mathrm{a}}$ of 
Table 1. Comparison of sensor characteristics for $\mathrm{pH}$ and oxygen sensors fabricated by hemiwicking (this work) and dip-coating.

\begin{tabular}{lcc}
\hline & This work & Dip-coating [3, 4] \\
\hline $\mathrm{p} K_{\mathrm{a}}$ & $6.43 \pm 0.08$ & $6.28 \pm 0.02$ \\
$\tau_{0}[\mu \mathrm{s}]$ & $6.061 \pm 0.024$ & $4.91 \pm 10 \%$ \\
$K_{\mathrm{SV}}\left[\mathrm{O}_{2} \%\right]^{-1}$ & $0.080 \pm 0.002$ & $0.072 \pm 0.002$ \\
\hline
\end{tabular}

$6.28 \pm 0.02$ given in [3], which was made using five different films produced by dip-coating. The small error bars in figure 9 indicate good spot-to-spot reproducibility.

Equally, on the oxygen sensor spots, excited-state life times $\tau$ were measured on 38 different spots in oxygen-free atmosphere $\tau_{0}$ and ambient air of $20.9 \%$ oxygen. The SternVolmer constant $K_{\mathrm{SV}}=\left(\left(\tau_{0} / \tau\right)-1\right) /\left[\mathrm{O}_{2} \%\right]$, where $\left[\mathrm{O}_{2} \%\right]$ is the concentration of $\mathrm{O}_{2}$ in percentages, was calculated.

In table 1 , the results of the sensor characterization are tabulated together with the results obtained in [3] and [4] on dip-coated films. The excited-state life time in oxygen-free atmosphere $\tau_{0}$ is seen to be larger than for the dip-coated sensors, which is most likely caused by the ten-fold thicker layer of the sensor material deposited on the microstructured surface. The thicker material layer may also explain the slightly larger value of $K_{\mathrm{SV}}$. However, it is worth noting that the standard deviation of $\tau_{0}$ is only $0.40 \%$ compared to the much larger $10 \%$ stated in [4]. The standard deviations of $K_{\mathrm{SV}}$ are of equal magnitude for the two deposition methods.

\section{Conclusion}

In conclusion, we have shown that hemiwicking can be used to spread a liquid sol-gel sensor material on a microstructured polycarbonate surface, and that, after evaporation of the solvents, the condensed material is uniformly distributed over the structured surface. The sensing performance of sensor spots fabricated by hemiwicking was characterized and found to be in good correspondence with the performance of sensors fabricated by dip-coating of the same sensor material. We have thus demonstrated an improved method for fabricating cheap optical sensors that can be integrated in disposable lab ware.

\section{References}

[1] McDonagh C, Burke C S and MacCraith B D 2008 Optical chemical sensors Chem. Rev. 108 400-22

[2] Naciri M, Kuystermans D and Al Rubeai M 2008 Monitoring $\mathrm{pH}$ and dissolved oxygen in mammalian cell culture using optical sensors Cytotechnology 57 245-50

[3] High performance optical ratiometric sol-gel-based $\mathrm{pH}$ sensor 2009 Sensors Actuators B 139 208-13

[4] Higgins C, Wencel D, Burke C S, MacCraith B D and McDonagh C 2008 Novel hybrid optical sensor materials for in-breath $\mathrm{O}_{2}$ analysis Analyst 133 241-7

[5] Brinker C J, Frye G C, Hurd A J and Ashley C S 1991 Fundamentals of sol-gel dip coating Thin Solid Films 201 97-108

[6] Hwang D K, Moon J H, Shul Y G, Jung K T, Kim D H and Lee D W 2003 Scratch resistant and transparent UV-protective coating on polycarbonate J. Sol-Gel Sci. Technol. 26 783-7

[7] Huang Y-Y and Chou K-S 2003 Studies on the spin coating process of silica films Ceram. Int. 29 485-93

[8] Quéré D 2008 Wetting and roughness Annu. Rev. Mater. Res. 38 71-99

[9] Haatainen T and Ahopelto J 2003 Pattern transfer using step and stamp imprint lithography Phys. Scr. 67357

[10] Utko P, Persson F, Kristensen A and Larsen N B 2011 Injection molded nanofluidic chips: fabrication method and functional tests using single-molecule DNA experiments Lab Chip 11 303-8

[11] Bico J, Thiele U and Quéré D 2002 Wetting of textured surfaces Colloids Surf. 206 41-6

[12] Bico J, Tordeux C and Quéré D 2001 Rough wetting Europhys. Lett. 55214

[13] Courbin L, Denieul E, Dressaire E, Roper M, Ajdari A and Stone H A 2007 Imbibition by polygonal spreading on microdecorated surfaces Nature Mater. 6 661-4

[14] Extrand C W, Moon S I, Hall P and Schmidt D 2007 Superwetting of structured surfaces Langmuir 23 8882-90

[15] Chang F-M, Hong S-J, Sheng Y-J and Tsao H-K 2010 Wetting invasion and retreat across a corner boundary J. Phys. Chem. C 114 1615-21

[16] Schift H 2008 Nanoimprint lithography: an old story in modern times? A review J. Vac. Sci. Technol. B 26 458-80

[17] Deegan R D, Bakajin O, Dupont T F, Huber G, Nagel S R and Witten T A 1997 Capillary flow as the cause of ring stains from dried liquid drops Nature 389 827-9

[18] Kimura S and Takami K 1988 Photoresist thickness measurement using laser-induced fluorescence Appl. Opt. 27 3675-8

[19] Pieper S B, Mestas S P, Lear K L, Zhong Z and Reardon K F 2008 Phosphorescence characteristics of ruthenium complex as an optical transducer for biosensors Appl. Phys. Lett. 92081915 